\title{
Sequential estimation of the parameters in unstable $\mathrm{AR}(2)$. $^{*}$
}

\author{
L. Galtchouk \\ IRMA, Department of Mathematics, \\ Strasbourg University \\ 7 , st. Réne Descartes \\ Strasbourg Cedex France \\ e-mail: galtchou@math.u-strasbg.fr \\ and \\ V.Konev \\ Department of Applied Mathematics and Cybernetics, \\ Tomsk University \\ Lenin str. 36, 634050 Tomsk, Russia \\ e-mail:vvkonev@vmm.tsu.ru
}

\begin{abstract}
For a second order non-explosive autoregressive process with unknown vector parameter $\theta=\left(\theta_{1}, \theta_{2}\right)^{\prime}$, it is shown that the sequential least squares estimate with a particular stopping time is asymptotically normally distributed uniformly in $\theta$ belonging to any compact set in the stability region of the process supplemented with the part of its boundary corresponding to complex roots of the characteristic polynomial.
\end{abstract}

AMS 1991 Subject Classification : 62L10, 62L12.

Key words and phrases: Autoregressive process, least squares estimator, sequential estimation, uniform asymptotic normality.

${ }^{*}$ This research was carried out at the Department of Mathematics, the Strasbourg University, France. The second author is partially supported by the RFFI-DFG-Grant 02-01-04001. 


\section{Introduction}

Consider the autoregressive $A R(2)$ model

$$
x_{n}=\theta_{1} x_{n-1}+\theta_{2} x_{n-2}+\varepsilon_{n}, n=1,2, \ldots,
$$

where $\left(x_{n}\right)$ is the observation, $\left(\varepsilon_{n}\right)$ is a sequence of independent identically distributed (i.i.d.) random variables with $\mathbf{E} \varepsilon_{1}=0$ and $0<\mathbf{E} \varepsilon_{1}^{2}=\sigma^{2}<\infty$, $\sigma^{2}$ is known, $x_{0}=x_{-1}=0$. The process (1.1) is assumed to be unstable, that is both roots of the characteristic polynomial

$$
\mathcal{P}(z)=z^{2}-\theta_{1} z-\theta_{2}
$$

lie on or inside the unit circle. The model (1.1) is a particular case of unstable autoregressive process $A R(p)$ which have been studied by many authors due to their applications in automatic control, identification and in modeling economic and financial time series (we refer the reader to Anderson (1971), Ahtola and Tiao (1987), Dickey and Fuller (1979), Chan and Wei (1988), Rao (1978) for details and futher references).

A commonly used estimate of parameter vector $\theta=\left(\theta_{1}, \theta_{2}\right)^{\prime}$ is the least squares estimate (LSE)

$$
\theta(n)=\left(\theta_{1}(n), \theta_{2}(n)\right)^{\prime}=M_{n}^{-1} \sum_{k=1}^{n} X_{k-1} x_{k}, \quad M_{n}=\sum_{k=1}^{n} X_{k-1} X_{k-1}^{\prime}
$$

where $X_{k}=\left(x_{k}, x_{k-1}\right)^{\prime} ;$ the prime denotes the transposition; $M_{n}^{-1}$ denotes the inverse of matrix $M_{n}$ if $\operatorname{det} M_{n}>0$ and $M_{n}^{-1}=0$ otherwise.

It is well known that

$$
\sqrt{n}(\theta(n)-\theta) \stackrel{\mathcal{L}}{\Longrightarrow} \mathcal{N}(0, F), \text { as } n \rightarrow \infty,
$$

for all $\theta \in \Lambda$, where $\Lambda$ is the stability region of process (1.1), that is

$$
\Lambda=\left\{\theta=\left(\theta_{1}, \theta_{2}\right)^{\prime}:-1+\theta_{2}<\theta_{1}<1-\theta_{2},\left|\theta_{2}\right|<1\right\}
$$

$F=F(\theta)$ is a positive definite matrix (see, e.g. Anderson (1971), Th. 5.5.7), $\stackrel{\mathcal{L}}{\Longrightarrow}$ indicates convergence in law. If $\theta$ belongs to the boundary $\partial \Lambda$ of the stability region $\Lambda$, the limiting distribution is no longer normal. Moreover, there is no one universal limiting distribution for all $\theta \in \partial \Lambda$ and the corresponding set of limiting distributions counts 6 different types depending on the values of roots $z_{1}$ and $z_{2}$ of polynomial (1.2). Each of the limiting distribution coincides with that of the ratio of certain Brownian functionals 
(see Chan and Wei (1988) for general results and details). For example, for a pair of conjugate complex roots $z_{1}=e^{i \varphi}, z_{2}=e^{-i \varphi}$ one has

$$
\begin{gathered}
n \cdot\left(\theta_{1}(n)-2 \cos \varphi\right) \stackrel{\mathcal{L}}{\Longrightarrow} \frac{\left(W_{1}^{2}(1)-W_{2}^{2}(1)\right) \sin \varphi+\left(W_{1}^{2}(1)+W_{2}^{2}(1)-2\right) \cos \varphi}{\int_{0}^{1}\left[W_{1}^{2}(s)+W_{2}^{2}(s)\right] d s}, \\
n \cdot\left(\theta_{2}(n)+1\right) \stackrel{\mathcal{L}}{\Longrightarrow}\left(2-W_{1}^{2}(1)-W_{2}^{2}(1)\right) / \int_{0}^{1}\left[W_{1}^{2}(s)+W_{2}^{2}(s)\right] d s,
\end{gathered}
$$

where $\left(W_{1}(t), 0 \leq t \leq 1\right)$ and $\left(W_{2}(t), 0 \leq t \leq 1\right)$ are independent standard Brownian motion processes. It is well-known that a similar situation takes place in case of $A R(1)$ process

$$
x_{n}=\theta x_{n-1}+\varepsilon_{n},
$$

for which the limiting distributions of the least squares estimate are not normal at the end-points $\theta= \pm 1$ of stability interval $(-1,1)$ (see, e.g. White (1958)).

Lai and Siegmund (1983) for a first order non-explosive autoregressive process proposed to use a sequential sampling scheme and proved that the sequential least squares estimate for $\theta$ with the stopping time based on the observed Fisher information is asymptotically normal uniformly in $\theta \in[-1,1]$ in contrast with the usual LSE.

In this paper we apply a sequential sampling scheme for estimating parameter vector $\theta=\left(\theta_{1}, \theta_{2}\right)^{\prime}$ in (1.1). The sequential least squares estimate is defined by replacing sample size $n$ in (1.3) with the stopping time

$$
\tau(h)=\inf \left\{n \geq 1: \sum_{k=1}^{n}\left(x_{k-1}^{2}+x_{k-2}^{2}\right) \geq h\right\}, \inf \{\emptyset\}=+\infty,
$$

where $h$ is a positive number (threshold). Our main result (Theorems 2.1) claims that as $h \rightarrow \infty$

$$
M_{\tau(h)}^{1 / 2}(\theta(\tau(h))-\theta) \stackrel{\mathcal{L}}{\longrightarrow} \mathcal{N}\left(0, \sigma^{2} I\right), I=\left(\begin{array}{ll}
1 & 0 \\
0 & 1
\end{array}\right)
$$

uniformly in $\theta \in K, K$ is any compact set in the stability region (1.4) supplemented with the part of its boundary

$$
\left\{\theta=\left(\theta_{1}, \theta_{2}\right)^{\prime}:-1+\theta_{2}<\theta_{1}<1-\theta_{2}, \theta_{2}=-1\right\},
$$

It will be observed that this part of the boundary includes the values of $\theta$ corresponding to the case when roots of polynomial (1.2) are $e^{i \phi}$ and $e^{-i \phi}$. 
Note that for stable autoregressive processes of order 1 with two unknown parameters and of order $p, p \geq 1$, the uniform asymptotic normality have been proved in author's papers $\left(2003_{a}, 2003_{b}\right)$.

The remainder of this paper is arranged as follows. Section 2 gives proofs of (1.6) and some properties of stopping time (1.5). In Section 3 some properties of unstable $A R(2)$ process needed to prove the main results are established. The appendix contains some technical results.

\section{Sequential least squares estimate. Uniform asymptotic normality.}

In this section we consider the sequential least squares estimate

$$
\begin{gathered}
\theta(\tau(h))=M_{\tau(h)}^{-1} \sum_{k=1}^{\tau(h)} X_{k-1} x_{k}, \\
\tau(h)=\inf \left\{n \geq 1: \sum_{k=1}^{n}\left\|X_{k-1}\right\|^{2} \geq h\right\}, \inf \{\emptyset\}=+\infty,
\end{gathered}
$$

and study its properties. First we will establish the property of uniform asymptotic normality (1.6).

Theorem 2.1 Let $\left(\varepsilon_{n}\right)_{n \geq 1}$ be a sequence of independent identically distributed random variables with $\mathbf{E} \varepsilon_{n}=0$ and $0<\mathbf{E} \varepsilon_{n}^{2}=\sigma^{2}<\infty$. Then for any compact set $K \subset \Lambda_{1}$

$$
\lim _{h \rightarrow \infty} \sup _{\theta \in K} \sup _{t \in R^{2}}\left|\mathbf{P}_{\theta}\left(M_{\tau(h)}^{1 / 2}(\theta(\tau(h))-\theta) \leq t\right)-\Phi_{2}(t / \sigma)\right|=0,
$$

where $\Phi_{2}(t)=\Phi\left(t_{1}\right) \Phi\left(t_{2}\right), \Phi$ is the standard normal distribution function,

$$
\Lambda_{1}=\left\{\theta=\left(\theta_{1}, \theta_{2}\right)^{\prime}:-1+\theta_{2}<\theta_{1}<1-\theta_{2},-1 \leq \theta_{2}<1\right\} .
$$

Proof. Substituting (1.1) in (2.1) yields

$$
M_{\tau(h)}^{1 / 2}\left(\theta(\tau(h)-\theta)=M_{\tau(h)}^{-1 / 2} \sum_{k=1}^{\tau(h)} X_{k-1} \varepsilon_{k}=\sqrt{h} M_{\tau(h)}^{-1 / 2} L^{1 / 2}\left(\theta_{1}, \theta_{2}\right) Y_{h}\right.
$$

where $L\left(\theta_{1}, \theta_{2}\right)$ is given in Lemma 3.4 ,

$$
Y_{h}=\frac{1}{\sqrt{h}} \sum_{k=1}^{\tau(h)} L^{-1 / 2}\left(\theta_{1}, \theta_{2}\right) X_{k-1} \varepsilon_{k}
$$

To prove Theorem 2.1 it suffices to establish the following Lemmas. 
Lemma 2.1 Under conditions of Theorem 2.1 for any compact set $K \subset \Lambda_{1}$ and $\delta>0$

$$
\lim _{h \rightarrow \infty} \sup _{\theta \in K} \mathbf{P}_{\theta}\left(\left\|\sqrt{h} M_{\tau(h)}^{-1 / 2} L^{1 / 2}\left(\theta_{1}, \theta_{2}\right)-I_{2}\right\|>\delta\right)=0,
$$

where $I_{2}$ is the unit matrix of order 2.

Lemma 2.2 Under conditions of Theorem 2.1 for any compact set $K \subset \Lambda_{1}$ and for each constant vector $v \in R^{2}$ with $\|v\|=1$

$$
\lim _{h \rightarrow \infty} \sup _{\theta \in K} \sup _{t \in R}\left|\mathbf{P}_{\theta}\left(v^{\prime} Y_{h} \leq t\right)-\Phi(t)\right|=0 .
$$

The proofs of these results are given in the Appendix.

By Lemma 2.2 vector $Y_{h}$ in $(2.4)$ is asymptotically normal uniformly in $\theta \in K$. In view of Lemma 2.1 this completes the proof of Theorem 2.1.

Now we will study the properties of the stopping time $\tau(h)$ defined by $(2.2)$. Denote

$$
\begin{gathered}
\Gamma_{1}=\left\{\theta=\left(\theta_{1}, \theta_{2}\right):-\theta_{1}+\theta_{2}=1,-2<\theta_{1}<0\right\}, \\
\Gamma_{2}=\left\{\theta=\left(\theta_{1}, \theta_{2}\right): \theta_{1}+\theta_{2}=1,0<\theta_{1}<2\right\}, \\
\Gamma_{3}=\left\{\theta=\left(\theta_{1},-1\right):-2<\theta_{1}<2\right\} \\
A=\left(\begin{array}{cc}
\theta_{1} & \theta_{2} \\
1 & 0
\end{array}\right) ; B=\left(\begin{array}{cc}
\sigma^{2} & 0 \\
0 & 0
\end{array}\right) .
\end{gathered}
$$

Theorem 2.2 Let $\left(\varepsilon_{n}\right)_{n \geq 1}$ in (1.1) be a sequence of independent identically distributed random variables with $E \varepsilon_{n}=0, E \varepsilon_{n}^{2}=\sigma^{2}$ and $\tau(h)$ be defined by (2.2). Let $z_{1}$ and $z_{2}$ be roots of polynomial (1.2). Then

for each $\theta \in \Lambda$

$$
\mathbf{P}_{\theta}-\lim _{h \rightarrow \infty} \frac{\tau(h)}{h}=\frac{1}{\operatorname{tr} F}, F-A F A^{\prime}=B ;
$$

for each $\theta \in \Gamma_{1}$

$$
\frac{\tau(h)}{\left(1+z_{2}\right) \sqrt{h / 2}} \stackrel{\mathcal{L}}{\Longrightarrow} \nu_{1}, \text { as } h \rightarrow \infty, \nu_{1}=\inf \left\{t \geq 0: \int_{0}^{t} W^{2}(s) d s=1\right\},
$$

for each $\theta \in \Gamma_{2}$

$$
\frac{\tau(h)}{\left(1-z_{1}\right) \sqrt{h / 2}} \stackrel{\mathcal{L}}{\Longrightarrow} \nu_{2}, \text { as } h \rightarrow \infty, \nu_{2}=\inf \left\{t \geq 0: \int_{0}^{t} W_{1}^{2}(s) d s=1\right\} \text {, }
$$


for each $\theta=(2 \cos \varphi,-1) \in \Gamma_{3}$

(2.8)

$\frac{\tau(h)}{\sqrt{2 h} \sin \varphi} \stackrel{\mathcal{L}}{\Longrightarrow} \nu_{3}$, as $h \rightarrow \infty, \nu_{3}=\inf \left\{t \geq 0: \int_{0}^{t}\left(W^{2}(s)+W_{1}^{2}(s)\right) d s \geq 1\right\}$,

where $W(t)$ and $W_{1}(t)$ are independent standard Brownian motions, $\Lambda$ is defined in (1.4).

Proof Assertion (2.5) easily follows from Lemma 3.12 in Galtchouk and Konev $\left(2003_{b}\right)$.

Let $\theta \in \Gamma_{1}$. In this case polynomial (1.2) takes the form

$$
\mathcal{P}(z)=(z+1)(z-a)
$$

where $-1<a<1$. By applying the backshift operator $q^{-1}$ one can write down equation (1.1) as

$$
q^{-2}(q+1)(q-a) x_{k}=\varepsilon_{k} .
$$

Denote

$$
u_{k}=q^{-1}(q-a) x_{k}, v_{k}=q^{-1}(q+1) x_{k},
$$

that is

$$
x_{k}-a x_{k-1}=u_{k}, x_{k}+x_{k-1}=v_{k},
$$

or in the vector form

$$
Q X_{k}=\left(\begin{array}{c}
u_{k} \\
v_{k}
\end{array}\right) ; Q=\left(\begin{array}{cc}
1 & -a \\
1 & 1
\end{array}\right)
$$

The processes $u_{k}$ and $v_{k}$ satisfy the equations

$$
u_{k}=-u_{k-1}+\varepsilon_{k}, v_{k}=a v_{k-1}+\varepsilon_{k}, u_{0}=0, v_{0}=0 \text {. }
$$

From here one gets

$$
u_{k}=-\sum_{j=1}^{k}(-1)^{j} \varepsilon_{j}, v_{k}=\sum_{j=1}^{k} a^{k-j} \varepsilon_{j} .
$$

The identity

$$
\sum_{k=1}^{n} x_{k-1}^{2}=(1 / 2) \sum_{k=1}^{n}\left\|X_{k-1}\right\|^{2}+\frac{x_{n-1}^{2}}{2}
$$


and (2.9) imply

(2.11)

$\sum_{k=1}^{n} x_{k-1}^{2}=\frac{1}{2} x_{n-1}^{2}+\frac{1}{(a+1)^{2}} \sum_{k=1}^{n} u_{k-1}^{2}+\frac{a-1}{a+1} \sum_{k=1}^{n} u_{k-1} v_{k-1}+\frac{a^{2}+1}{2(a+1)^{2}} \sum_{k=1}^{n} v_{k-1}^{2}$.

It is known that (Lai and Wei (1983), Chan and Wei (1988))

$$
\begin{gathered}
\liminf _{n \rightarrow \infty} \frac{\sum_{k=1}^{n} u_{k-1}^{2}}{n^{2} / \log \log n}=\sigma^{2} / 4 \quad \mathbf{P}_{\theta}-\text { a.s. } \\
P_{\theta}-\lim _{n \rightarrow \infty} n^{-3 / 2} \sum_{k=1}^{n} u_{k-1} v_{k-1}=0 .
\end{gathered}
$$

This and (2.11) yield

$$
P_{\theta}-\lim _{n \rightarrow \infty} \frac{\sum_{k=1}^{n} x_{k-1}^{2}}{\sum_{k=1}^{n} u_{k-1}^{2}}=(a+1)^{-2}
$$

Further we have

$$
P_{\theta}\left(\frac{\tau(h)}{b \sqrt{h}} \leq t\right)=P_{\theta}\left(\sum_{k=1}^{[t b \sqrt{h}]}\left\|X_{k-1}\right\|^{2} \geq h\right)=P_{\theta}\left(2 \sum_{k=1}^{[t b \sqrt{h}]} x_{k-1}^{2}-x_{[t b \sqrt{h}]-1} \geq h\right)
$$

where $b=(a+1) / \sqrt{2}$. Applying herein Lemma 3.2 and (2.10) yields

$$
P_{\theta}\left(\frac{\tau(h)}{b \sqrt{h}} \leq t\right)=P_{\theta}\left(\frac{1}{b^{2} h} \sum_{k=1}^{[t b \sqrt{h}]} u_{k-1}^{2} \mu_{[t b \sqrt{h}]} \geq 1\right)
$$

where $P_{\theta}-\lim _{h \rightarrow \infty} \mu_{[t b \sqrt{h}]}=1$. From here by Donsker's Theorem we come to (2.6). By a similar argument one can show (2.7).

Assume that $\theta \in \Gamma_{3}$. Then in view of $(2.10)$ one has (2.13)

$$
P_{\theta}\left(\frac{\tau(h)}{b h^{1 / 2}} \leq t\right)=P_{\theta}\left(\sum_{k=1}^{\left[t b h^{1} / 2\right]}\left\|X_{k-1}\right\|^{2} \geq h\right)=P_{\theta}\left(2 b^{2} Z_{\left[t b h^{1 / 2}\right]} \tilde{\mu}_{[t b \sqrt{h}]} \geq 1\right),
$$

where

$$
Z_{[t b \sqrt{h}]}=\frac{1}{b^{2} h} \sum_{k=1}^{\left[t b h^{1} / 2\right]} x_{k-1}^{2}
$$

and

$$
P_{\theta}-\lim _{h \rightarrow \infty} \tilde{\mu}_{[t b \sqrt{h}]}=1
$$


Now we apply Theorem (3.3.4) from the paper of Chan and Wei (1988), which yields

$$
Z_{[t b \sqrt{h}]} \stackrel{\mathcal{L}}{\Longrightarrow} \frac{1}{4 \sin ^{2} \phi} \int_{0}^{t}\left(W^{2}(s)+W_{1}^{2}(s)\right) d s .
$$

Putting $b=\sqrt{2} \sin \phi$ in (2.13) and taking this into account we come to (2.8). This completes the proof.

\section{Auxiliary propositions.}

In this section we establish some properties of the process (1.1) and the observed Fisher information matrix $M_{n}$.

In the sequel we will need the following two probabilistic results for martingales from the paper of Lai and Siegmund (1983).

Proposition 3.1 Let $x_{n}, \varepsilon_{n}, n=0,1, \ldots$ be random variables adapted to the increasing sequence of $\sigma$-algebras $\left(\mathcal{F}_{n}\right)_{n \geq 0}$. Let $\left\{\mathbf{P}_{\theta}, \theta \in \Theta\right\}$ be a family of probability measures such that under every $\mathbf{P}_{\theta}$

$A_{1}: \varepsilon_{1}, \varepsilon_{2}, \ldots$ are i.i.d. with $\mathbf{E}_{\theta} \varepsilon_{1}=0, \mathbf{E}_{\theta} \varepsilon_{1}^{2}=1$;

$A_{2}: \sup _{\theta} \mathbf{E}_{\theta}\left\{\varepsilon_{1}^{2} ;\left|\varepsilon_{1}\right|>a\right\} \rightarrow 0$ as $a \rightarrow \infty$;

$A_{3}: \varepsilon_{n}$ is independent of $\mathcal{F}_{n-1}$ for each $n \geq 1$;

$A_{4}: \mathbf{P}_{\theta}\left(\sum_{i=0}^{\infty} x_{i}^{2}=\infty\right)=1$;

$A_{5}: \sup _{\theta} \mathbf{P}_{\theta}\left(x_{n}^{2}>a\right) \rightarrow 0$ as $a \rightarrow \infty$ for each $n \geq 0$;

$A_{6}: \lim _{m \rightarrow \infty}\left[\sup _{\theta} \mathbf{P}_{\theta}\left(x_{n}^{2} \geq \delta \sum_{i=0}^{n-1} x_{i}^{2}\right.\right.$ for some $\left.\left.n \geq m\right)\right]=0$ for each $\delta>0$.

For $c>0$ let $T_{c}=\inf \left\{n: \sum_{i=1}^{n} x_{i-1}^{2} \geq c\right\}, \inf \{\emptyset\}=+\infty$. Then uniformly in $\theta \in \Theta$ and $-\infty<t<\infty$

$$
\mathbf{P}_{\theta}\left\{c^{-1 / 2} \sum_{i=1}^{T_{c}} x_{i-1} \varepsilon_{i} \leq t\right\} \rightarrow \Phi(t) \text { as } c \rightarrow \infty,
$$

where $\Phi$ is the standard normal distribution function.

Lemma 3.1 Suppose that the measurability conditions of Proposition 3.1, $A_{1}, A_{3}$ are satisfied. Then for each $\gamma>1 / 2, \delta>0$, and increasing sequence of positive constants $c_{n} \rightarrow \infty$,

$$
\sup _{\theta \in \Theta} \mathbf{P}_{\theta}\left\{\left|\sum_{k=1}^{n} x_{k-1} \varepsilon_{k}\right| \geq \delta \max \left(c_{n},\left(\sum_{k=1}^{n} x_{k-1}^{2}\right)^{\gamma}\right) \text { for some } n \geq m\right\} \rightarrow 0
$$

as $m \rightarrow \infty$. 
Now we show the following result.

Lemma 3.2 Let $\left(\varepsilon_{n}\right)_{n \geq 1}$ in (1.1) be a sequence of i.i.d. random variables with $\mathbf{E} \varepsilon_{n}=0$ and $\mathbf{E} \varepsilon_{n}^{2}=\sigma^{2}<\infty$ and roots of the characteristic polynomial (1.2) lie on or inside the unit circle, i.e. $\left|z_{1}\right| \leq 1,\left|z_{2}\right| \leq 1$. Then, for any compact set $K \subset \Lambda_{0}$ and $\delta>0$,

$$
\lim _{m \rightarrow \infty} \sup _{\theta \in K} \mathbf{P}_{\theta}\left(\max \left(x_{n}^{2}, x_{n-1}^{2}\right) \geq \delta \sum_{k=1}^{n} x_{k-1}^{2} \text { for some } n \geq m\right)=0,
$$

where

$$
\Lambda_{0}=[\Lambda] \backslash\{(-2,-1),(2,-1)\},
$$

$[\Lambda]$ is the closure of the stability region (1.4).

Proof. In view of the equality

$$
\sum_{k=1}^{n} x_{k-1}^{2}=(1 / 2) \sum_{k=1}^{n}\left\|X_{k-1}\right\|^{2}+\frac{x_{n-1}^{2}}{2}
$$

it suffices to show that, for any compact set $K \subset \Lambda_{0}$ and $\delta>0$,

$$
\lim _{m \rightarrow \infty} \sup _{\theta \in K} \mathbf{P}_{\theta}\left(B_{m}(\delta)\right)=0
$$

where

$$
B_{m}(\delta)=\left\{\left\|X_{n}\right\|^{2} \geq \delta \sum_{k=1}^{n}\left\|X_{k-1}\right\|^{2} \text { for some } n \geq m\right\} .
$$

By making use of the vector form of equation (1.1)

$$
X_{n}=A X_{n-1}+\xi_{n}
$$

where $X_{n}=\left(x_{n}, x_{n-1}\right)^{\prime}, \xi_{n}=\left(\varepsilon_{n}, 0\right)^{\prime}$,

$$
A=\left(\begin{array}{cc}
\theta_{1} & \theta_{2} \\
1 & 0
\end{array}\right), \xi_{n}=\left(\begin{array}{c}
\varepsilon_{n} \\
0
\end{array}\right) .
$$

one obtains

$$
\sum_{k=1}^{n} X_{k-1} X_{k-1}^{\prime} \geq \sum_{k=1}^{n} \xi_{k} \xi_{k}^{\prime}-X_{n} X_{n}^{\prime}+\sum_{k=1}^{n} \xi_{k} X_{k-1}^{\prime} A^{\prime}+A \sum_{k=1}^{n} X_{k-1} \xi_{k}
$$


This implies

$$
\sum_{k=1}^{n}\left\|X_{k-1}\right\|^{2} \geq \sum_{k=1}^{n} \varepsilon_{k}^{2}-\left\|X_{n}\right\|^{2}-2\left|\sum_{k=1}^{n}<A X_{k-1}>_{1} \varepsilon_{k}\right|,
$$

where $<A X_{k-1}>_{1}$ is the first coordinate of vector $A X_{k-1}$.

For each $0<\lambda<\sigma^{2} / 4$ and $m \geq 1$ we introduce the sets

$$
\begin{gathered}
\Omega_{m, \lambda}=\left\{\left|n^{-1} \sum_{k=1}^{n} \varepsilon_{k}^{2}-\sigma^{2}\right|<\lambda\right\} \cap \\
\left\{\left|\sum_{k=1}^{n}<A X_{k-1}>_{1} \varepsilon_{k}\right|<\max \left(\lambda n,\left(\sum_{k=1}^{n}<A X_{k-1}>_{1}^{2}\right)^{2 / 3}\right) \text { for all } n \geq m\right\} .
\end{gathered}
$$

It is easily seen that

$$
\Omega_{m, \lambda} \subset\left\{\sum_{k=1}^{m}\left\|X_{k-1}\right\|^{2} \leq 1\right\} \bigcap \Omega_{m, \lambda}^{\prime}
$$

where

$$
\begin{gathered}
\Omega_{m, \lambda}^{\prime} \subset \cap_{n \geq m}\left(\mathcal{D}_{n} \cup\left\{\left\|X_{n}\right\|^{2}>\lambda n\right\}\right), \\
\mathcal{D}_{n}=\left\{\left|n^{-1} \sum_{k=1}^{n} \varepsilon_{k}^{2}-\sigma^{2}\right|<\lambda\right\} \cap \\
\left\{\left|\sum_{k=1}^{n}<A X_{k-1}>_{1} \varepsilon_{k}\right|<\max \left(\lambda n,\left(\sum_{k=1}^{n}<A X_{k-1}>_{1}^{2}\right)^{2 / 3}\right),\right. \\
\left.\left\|X_{n}\right\|^{2} \leq \lambda n, \sum_{k=1}^{m}\left\|X_{k-1}\right\|^{2}>1\right\} .
\end{gathered}
$$

By (3.6), on the set $\mathcal{D}_{n}$, one has the inequality

$$
\begin{gathered}
\sum_{k=1}^{n}\left\|X_{k-1}\right\|^{2} \geq\left(\sigma^{2}-\lambda\right) n-\lambda n-2 \lambda n-2\left(\sum_{k=1}^{n}\left\|A X_{k-1}\right\|^{2}\right)^{2 / 3} \\
\geq\left(\sigma^{2}-4 \lambda\right) n-2\|A\|^{4 / 3}\left(\sum_{k=1}^{n}\left\|X_{k-1}\right\|^{2}\right)^{2 / 3}
\end{gathered}
$$


which implies

$$
\left(1+2\|A\|^{4 / 3}\right) \sum_{k=1}^{n}\left\|X_{k-1}\right\|^{2} \geq\left(\frac{\sigma^{2}}{\lambda}-4\right) \lambda n \geq\left(\frac{\sigma^{2}}{\lambda}-4\right)\left\|X_{n}\right\|^{2} .
$$

Given $\delta>0$, by choosing $\lambda$ so that

$$
\left(1+2 \sup _{\theta \in K}\|A\|^{4 / 3}\right)\left(\frac{\sigma^{2}}{\lambda}-4\right)^{-1}<\delta
$$

one has, on the set $\mathcal{D}_{n}$, the inequality

$$
\left\|X_{n}\right\|^{2}<\delta \sum_{k=1}^{n}\left\|X_{k-1}\right\|^{2}, n \geq m
$$

This and (3.8) lead to the inclusion

$$
\Omega_{m, \lambda}^{\prime} \subset \bigcap_{n \geq m}\left(\left\{\left\|X_{n}\right\|^{2}<\delta \sum_{k=1}^{n}\left\|X_{k-1}\right\|^{2}\right\} \bigcup\left\{\left\|X_{n}\right\|^{2}>\lambda n\right\}\right) .
$$

Now assume that $\left\|X_{n}\right\|^{2} \geq \lambda n$. By (3.4) one gets

$$
X_{n}=A X_{n-k}+\sum_{j=0}^{k-1} A^{j} \xi_{n-j}
$$

Further we will show that for every compact set $K \subset \Lambda_{0}$ there exists a positive number $\kappa$ such that

$$
\sup _{\theta \in K}\left\|A^{n}\right\| \leq \kappa, n \geq 1 .
$$

Without loss of generality we can consider a compact set of the form

$K_{d}=[-2+d, 2-d] \times[-1,-1+d] \cup\left\{\theta:-1+\theta_{2} \leq \theta_{1} \leq 1-\theta_{2},-1+d \leq \theta_{2} \leq 1\right\}$, where $0<d \leq 2$. One can verify that if roots $z_{1}$ and $z_{2}$ of the characteristic polynomial $(1.2)$ are real then

$$
A^{n}=\frac{1}{z_{1}-z_{2}}\left(\begin{array}{ll}
z_{1}^{n+1}-z_{2}^{n+1} & -\left(z_{1}^{n}-z_{2}^{n}\right) z_{1} z_{2} \\
z_{1}^{n}-z_{2}^{n} & -\left(z_{1}^{n-1}-z_{2}^{n-1}\right) z_{1} z_{2}
\end{array}\right), \quad-1 \leq z_{1}<z_{2} \leq 1
$$

and, if the roots are complex, that is $z_{1}=a e^{i \phi}$ and $z_{2}=a e^{i \phi}$, then

$$
A^{n}=\frac{a^{n-1}}{\sin \phi}\left(\begin{array}{ll}
\sin (n+1) \phi & -a^{2} \sin (n \phi) \\
\sin n \phi & -\sin (n-1) \phi
\end{array}\right) .
$$


By making use of these formulas respectively on the subsets

$$
\begin{gathered}
K_{d}^{\prime}=[-2+d, 2-d] \times[-1,-1+d] \cup\left\{\theta:-2+d \leq \theta_{1} \leq 2-d,-1 \leq \theta_{2} \leq-\frac{1}{4} \theta^{2}\right\}, \\
K_{d}^{\prime \prime}=\left\{\theta:-2+d \leq \theta_{1} \leq 0,-\frac{1}{4} \theta_{1}^{2}<\theta_{2} \leq 1+\theta_{1}\right\} \\
\bigcup\left\{\theta: 0 \leq \theta_{1} \leq 2-d,-\frac{1}{4} \theta_{1}^{2}<\theta_{2} \leq 1-\theta_{1}\right\},
\end{gathered}
$$

we establish that (3.9) is satisfied with $K=K_{d}$. From (3.10) in view of (3.11) one obtains

$$
\kappa\left\|X_{n-i}\right\| \geq\left\|A^{i}\right\| \cdot\left\|X_{n-i}\right\| \geq\left\|A^{i} X_{n-i}\right\| \geq\left\|X_{n}\right\|-\sum_{j=0}^{i-1}\left\|A^{j}\right\| \cdot\left|\varepsilon_{n-j}\right| .
$$

This implies that for $k \leq n$

$$
\begin{aligned}
& \kappa \min _{1 \leq i \leq k}\left\|X_{n-i}\right\| \geq\left\|X_{n}\right\|-\sum_{j=0}^{k-1}\left\|A^{j}\right\| \cdot\left|\varepsilon_{n-j}\right| \\
& \geq\left\|X_{n}\right\|-\zeta_{n, k} \geq\left\|X_{n}\right\|\left(1-(\lambda n)^{-1 / 2} \zeta_{n, k}\right),
\end{aligned}
$$

where

$$
\zeta_{n, k}=\kappa \sum_{j=0}^{k-1}\left|\varepsilon_{n-j}\right|
$$

Therefore

$$
\sum_{i=1}^{n}\left\|X_{i-1}\right\|^{2} \geq \sum_{i=1}^{n}\left\|X_{n-i}\right\|^{2} \geq\left\|X_{n}\right\|^{2} k\left(1-(\lambda n)^{-1 / 2} \zeta_{n, k}\right)^{2} / \kappa, k \leq n .
$$

This inequality yields the inclusions

$$
\left\{\left\|X_{n}\right\|^{2}>\lambda n\right\} \subset\left\{\sum_{i=1}^{n}\left\|X_{i-1}\right\|^{2} \geq\left\|X_{n}\right\|^{2} \frac{k}{\kappa}(1-\triangle)^{2}\right\} \cup\left\{\sup _{n \geq m}(\lambda n)^{-1 / 2} \zeta_{n, k}>\Delta\right\}
$$

for $n \geq m$, where $0<\Delta<1$. Choosing $k$ so that

$$
k(1-\Delta)^{2} / \kappa^{2}>\delta^{-1}
$$

we obtain

$$
\left\{\left\|X_{n}\right\|^{2}>\lambda n\right\} \supset\left\{\left\|X_{n}\right\|^{2}<\delta \sum_{k=1}^{n}\left\|X_{k-1}\right\|^{2}\right\} \subset\left\{\sup _{n \geq m}(\lambda n)^{-1 / 2} \zeta_{n, k}>\Delta\right\}, n \geq m .
$$


From here and (3.7), (3.9) it follows that

$$
\begin{gathered}
\Omega_{m, \lambda} \subset\left\{\sum_{k=1}^{m}\left\|X_{k-1}\right\|^{2} \leq 1\right\} \bigcup\left\{\sup _{n \geq m}(\lambda n)^{-1 / 2} \zeta_{n, k}>\Delta\right\} \\
\bigcup_{n \geq m}\left\{\left\|X_{n}\right\|^{2}<\delta \sum_{k=1}^{n}\left\|X_{k-1}\right\|^{2}\right\} .
\end{gathered}
$$

This implies

$$
\Omega_{m, \lambda}^{c} \supset\left\{\sum_{k=1}^{m}\left\|X_{k-1}\right\|^{2}>1\right\} \supset\left\{\sup _{n \geq m}(\lambda n)^{-1 / 2} \zeta_{n, k} \leq \Delta\right\} \supset B_{m}(\delta) .
$$

Finally we get the inclusion

$$
B_{m}(\delta) \supset \Omega_{m, \lambda}^{c} \bigcup\left\{\sum_{k=1}^{m}\left\|X_{k-1}\right\|^{2} \leq 1\right\} \bigcup\left\{\sup _{n \geq m} \frac{\zeta_{n, k}}{\sqrt{\lambda n}}>\Delta\right\} .
$$

Hence

$$
\begin{gathered}
\sup _{\Theta \in K} P_{\Theta}\left(B_{m}(\delta)\right) \leq \sup _{\Theta \in K} P_{\Theta}\left(\Omega_{m, \lambda}^{c}\right)+\sup _{\Theta \in K} P_{\Theta}\left\{\sum_{k=1}^{m}\left\|X_{k-1}\right\|^{2} \leq 1\right\} \\
+\sup _{\Theta \in K} P_{\Theta}\left\{\sup _{n \geq m}(\lambda n)^{-1 / 2} \zeta_{n, k}>\Delta\right\} .
\end{gathered}
$$

By Lemma 3.1 with $x_{i-1}=\left\langle A X_{i-1}\right\rangle_{1}$ and $\gamma=2 / 3$ the first term in the righthand side of this inequality vanishes as $m \rightarrow \infty$. The last two summands tend to zero by the law of large numbers.

This completes the proof of Lemma 3.2.

Lemma 3.3 Let $\left(\varepsilon_{n}\right)_{n \geq 1}$ in (1.1) be a sequence of i.i.d. random variables with $\mathbf{E} \varepsilon_{n}=0$ and $\mathbf{E} \varepsilon_{n}^{2}=\sigma^{2}<\infty$. Then for any compact set $K \subset \Lambda_{0}$

$$
\lim _{m \rightarrow \infty} \sup _{\theta \in K} \mathbf{P}_{\theta}\left(\left|\sum_{k=1}^{n} x_{k-1} \varepsilon_{k}\right|>\delta \sum_{k=1}^{n} x_{k-1}^{2} \text { for some } n \geq m\right)=0
$$

where $\Lambda_{0}$ is given in (3.1).

Proof. Let $c_{n}=n^{3 / 4}$. For the set of interest one has the following inclusions

$$
\left\{\left|\sum_{k=1}^{n} x_{k-1} \varepsilon_{k}\right|>\delta \sum_{k=1}^{n} x_{k-1}^{2} \text { for some } n \geq m\right\}
$$




$$
\begin{gathered}
=\left\{\frac{\left|\sum_{k=1}^{n} x_{k-1} \varepsilon_{k}\right|}{\left(\sum_{k=1}^{n} x_{k-1}^{2}\right)^{2 / 3} \vee c_{n}} \cdot \frac{\left(\sum_{k=1}^{n} x_{k-1}^{2}\right)^{2 / 3} \vee c_{n}}{\sum_{k=1}^{n} x_{k-1}^{2}}>\delta \text { for some } n \geq m\right\} \\
\subset\left\{\frac{\left|\sum_{k=1}^{n} x_{k-1} \varepsilon_{k}\right|}{\left(\sum_{k=1}^{n} x_{k-1}^{2}\right)^{2 / 3} \vee c_{n}}>\sqrt{\delta} \text { for some } n \geq m\right\} \cup \\
\quad\left\{\left(\sum_{k=1}^{n} x_{k-1}^{2}\right)^{-1 / 3} \vee c_{n}\left(\sum_{k=1}^{n} x_{k-1}^{2}\right)^{-1}>\sqrt{\delta} \text { for some } n \geq m\right\} \\
\subset\left\{\left|\sum_{k=1}^{n} x_{k-1} \varepsilon_{k}\right|\left(\left(\sum_{k=1}^{n} x_{k-1}^{2}\right)^{2 / 3} \vee c_{n}\right)^{-1}>\sqrt{\delta} \text { for some } n \geq m\right\} \cup \\
\left.\cup\left\{\left(\sum_{k=1}^{n} x_{k-1}^{2}\right)^{-1}>\delta^{3 / 2} \text { for some } n \geq m\right\} \cup\right\} \\
\cup\left\{c_{n}\left(\sum_{k=1}^{n} x_{k-1}^{2}\right)^{-1}>\sqrt{\delta} \text { for some } n \geq m\right\} \\
\subset\left\{\sum_{k=1}^{n} x_{k-1} \varepsilon_{k} \mid\left(\left(\sum_{k=1}^{n} x_{k-1}^{2}\right)^{2 / 3} \vee c_{n}\right)^{-1}>\sqrt{\delta} \text { for some } n \geq m\right\} \\
\cup\left\{c_{n}\left(\sum_{k=1}^{n} x_{k-1}^{2}\right)^{-1}>\sqrt{\delta} \wedge \delta^{3 / 2} \text { for some } n \geq m\right\} .
\end{gathered}
$$

From here it follows that

$$
\begin{gathered}
\mathbf{P}_{\theta}\left(\left|\sum_{k=1}^{n} x_{k-1} \varepsilon_{k}\right|>\delta \sum_{k=1}^{n} x_{k-1}^{2} \text { for some } n \geq m\right) \\
\leq \mathbf{P}_{\theta}\left\{\left|\sum_{k=1}^{n} x_{k-1} \varepsilon_{k}\right|\left(\left(\sum_{k=1}^{n} x_{k-1}^{2}\right)^{2 / 3} \vee c_{n}\right)^{-1}>\sqrt{\delta} \text { for some } n \geq m\right\} \\
+\mathbf{P}_{\theta}\left\{n^{3 / 4}\left(\sum_{k=1}^{n} x_{k-1}^{2}\right)^{-1}>\sqrt{\delta} \wedge \delta^{3 / 2} \text { for some } n \geq m\right\} .
\end{gathered}
$$

By making use of (1.1) and the elementary inequalities we obtain

$$
\sum_{k=1}^{n} \varepsilon_{k}^{2}=\sum_{k=1}^{n}\left(x_{k}-\theta_{1} x_{k-1}-\theta_{2} x_{k-2}\right)^{2}
$$




$$
\begin{gathered}
\leq 3\left(\sum_{k=1}^{n} x_{k}^{2}+\theta_{1}^{2} \sum_{k=1}^{n} x_{k-1}^{2}+\theta_{2}^{2} \sum_{k=1}^{n} x_{k-2}^{2}\right) \\
\leq 15\left(x_{n}^{2}+\sum_{k=1}^{n} x_{k-1}^{2}\right)=15 \sum_{k=1}^{n} x_{k-1}^{2}\left(1+\frac{x_{n}^{2}}{\sum_{k=1}^{n} x_{k-1}^{2}}\right) .
\end{gathered}
$$

Therefore the second summand in the right-hand side of (3.13) can be estimated as

$$
\begin{aligned}
& \mathbf{P}_{\theta}\left\{n^{3 / 4}\left(\sum_{k=1}^{n} x_{k-1}^{2}\right)^{-1}>\sqrt{\delta} \wedge \delta^{3 / 2} \text { for some } n \geq m\right\} \\
& \leq \mathbf{P}_{\theta}\left\{30 n^{3 / 4}\left(\sum_{k=1}^{n} \varepsilon_{k}^{2}\right)^{-1}>\sqrt{\delta} \wedge \delta^{3 / 2} \text { for some } n \geq m\right\} \\
& +\mathbf{P}_{\theta}\left\{x_{n}^{2} / \sum_{k=1}^{n} x_{k-1}^{2} \geq 1 \text { for some } n \geq m\right\} .
\end{aligned}
$$

Combining this and (3.13) and applying Lemmas 3.1, 3.2 lead to (3.12).

This completes the proof of Lemma 3.3.

Lemma 3.4 Let $\left(\varepsilon_{n}\right)_{n \geq 1}$ in (1.1) be a sequence of i.i.d. random variables with $\mathbf{E} \varepsilon_{n}=0$ and $\mathbf{E} \varepsilon_{n}^{2}=\sigma^{2}<\infty$ and $M_{n}$ be given by (1.3).

Then, for any compact set $K \subset \grave{\Lambda}$ and $\delta>0$,

$$
\lim _{m \rightarrow \infty} \sup _{\theta \in K} \mathbf{P}_{\theta}\left(\left\|\frac{M_{n}}{\sum_{k=1}^{n} x_{k-1}^{2}}-L\left(\theta_{1}, \theta_{2}\right)\right\| \geq \delta \text { for some } n \geq m\right)=0
$$

where

$$
L\left(\theta_{1}, \theta_{2}\right)=\left(\begin{array}{cc}
1 & \frac{\theta_{1}}{1-\theta_{2}} \\
\frac{\theta_{1}}{1-\theta_{2}} & 1
\end{array}\right), \stackrel{\circ}{\Lambda}=[\Lambda] \backslash\{(0,1),(-2,-1),(2,-1)\} .
$$

Proof. Multiplying (1.1) by $x_{k-1}$ and summing yield

$$
\sum_{k=1}^{n} x_{k-1} x_{k}=\theta_{1} \sum_{k=1}^{n} x_{k-1}^{2}+\theta_{2} \sum_{k=1}^{n} x_{k-1} x_{k-2}+\sum_{k=1}^{n} x_{k-1} \varepsilon_{k} .
$$

From here it follows that

$$
\sum_{k=1}^{n} x_{k-1} x_{k-2}=-\frac{1}{1-\theta_{2}} x_{n} x_{n-1}+\frac{\theta_{1}}{1-\theta_{2}} \sum_{k=1}^{n} x_{k-1}^{2}+\frac{1}{1-\theta_{2}} \sum_{k=1}^{n} x_{k-1} \varepsilon_{k} .
$$


Substituting this in $M_{n}$ yields

$$
M_{n}=L\left(\theta_{1}, \theta_{2}\right) \sum_{k=1}^{n} x_{k-1}^{2}+r_{n}
$$

where

$$
r_{n}=\frac{1}{1-\theta_{2}}\left(\begin{array}{cc}
0 ; & x_{n} x_{n-1}+\sum_{k=1}^{n} x_{k-1} \varepsilon_{k} \\
-x_{n} x_{n-1}+\sum_{k=1}^{n} x_{k-1} \varepsilon_{k} ; & -x_{n-1}^{2}
\end{array}\right) .
$$

By applying Lemmas $3.3,3.4$ to $r_{n} / \sum_{k=1}^{n} x_{k-1}^{2}$ we come to (3.14). This completes the proof of Lemma 3.4.

Lemma 3.5 Let $M_{n}, \tau(h)$ and $L\left(\theta_{1}, \theta_{2}\right)$ be given by (1.3), (1.5) and (3.15) respectively. Assume that $\left\{\varepsilon_{n}\right\}$ in (1.1) be a sequence of independent identically distributed random variables with $E \varepsilon_{n}=0, E \varepsilon_{n}=\sigma^{2}$. Then, for any compact set $K \subset \stackrel{\circ}{\Lambda}$ and $\delta>0$,

$$
\lim _{h \rightarrow \infty} \sup _{\theta \in K} \mathbf{P}_{\theta}\left(\left\|\frac{M_{\tau(h)}}{h}-L\left(\theta_{1}, \theta_{2}\right)\right\|>\delta\right)=0,
$$

where $\stackrel{\Lambda}{\Lambda}$ is the same as in (3.15).

Proof. By making use of the equality

$$
\frac{M_{\tau(h)}}{h}-L\left(\theta_{1}, \theta_{2}\right)=\frac{M_{\tau(h)}}{\sum_{k=1}^{\tau(h)} x_{k-1}^{2}}-L\left(\theta_{1}, \theta_{2}\right)+M_{\tau(h)}\left(\frac{1}{h}-\frac{1}{\sum_{k=1}^{\tau(h)} x_{k-1}^{2}}\right)
$$

one gets

$$
\left\|\frac{M_{\tau(h)}}{h}-L\left(\theta_{1}, \theta_{2}\right)\right\| \leq\left\|\frac{M_{\tau(h)}}{\sum_{k=1}^{\tau(h)} x_{k-1}^{2}}-L\left(\theta_{1}, \theta_{2}\right)\right\|+\frac{\left\|M_{\tau(h)}\right\|}{\sum_{k=1}^{\tau(h)} x_{k-1}^{2}} \frac{\left(\sum_{k=1}^{\tau(h)} x_{k-1}^{2}-h\right)}{h} .
$$

From here it follows that

$$
\begin{gathered}
\left\{\left\|\frac{M_{\tau(h)}}{h}-L\left(\theta_{1}, \theta_{2}\right)\right\|>\delta\right\} \subset\left\{\left\|\frac{M_{\tau(h)}}{\sum_{k=1}^{\tau(h)} x_{k-1}^{2}}-L\left(\theta_{1}, \theta_{2}\right)\right\|>\delta / 2\right\} \\
\cup\left\{\left\|\frac{M_{\tau(h)}}{\sum_{k=1}^{\tau(h)} x_{k-1}^{2}}\right\| \frac{\left(\sum_{k=1}^{\tau(h)} x_{k-1}^{2}-h\right)}{h}>\delta / 2\right\} .
\end{gathered}
$$


Further we have the inclusions

$$
\begin{gathered}
\left\{\left\|\frac{M_{\tau(h)}}{\sum_{k=1}^{\tau(h)} x_{k-1}^{2}}-L\left(\theta_{1}, \theta_{2}\right)\right\|>\delta / 2\right\} \subseteq\{\tau(h) \leq m\} \\
\cup\left\{\left\|\frac{M_{n}}{\sum_{k=1}^{n} x_{k-1}^{2}}-L\left(\theta_{1}, \theta_{2}\right)\right\|>\delta / 2 \text { for some } n \geq m\right\}, \\
\left\{\left\|\frac{M_{\tau(h)}}{\sum_{k=1}^{\tau(h)} x_{k-1}^{2}}\right\| \frac{\left(\sum_{k=1}^{\tau(h)} x_{k-1}^{2}-h\right)}{h}>\delta / 2\right\} \subset\{\tau(h) \leq m\} \\
\cup\left\{\frac{\left\|M_{n}\right\|}{\sum_{k=1}^{n} x_{k-1}^{2}} \cdot \frac{x_{n-1}^{2}}{\sum_{k=1}^{n-1} x_{k-1}^{2}}>\delta / 2 \text { for some } n \geq m\right\} .
\end{gathered}
$$

From here and (3.17) one obtains

$$
\begin{gathered}
\mathbf{P}_{\theta}\left\{\left\|\frac{M_{\tau(h)}}{h}-L\left(\theta_{1}, \theta_{2}\right)\right\|>\delta\right\} \leq 2 \mathbf{P}_{\theta}\{\tau(h) \leq m\} \\
+\mathbf{P}_{\theta}\left\{\left\|\frac{M_{n}}{\sum_{k=1}^{n} x_{k-1}^{2}}-L\left(\theta_{1}, \theta_{2}\right)\right\|>\delta / 2 \text { for some } n \geq m\right\} \\
+\mathbf{P}_{\theta}\left\{\frac{\left\|M_{n}\right\|}{\sum_{k=1}^{n} x_{k-1}^{2}} \cdot \frac{x_{n-1}^{2}}{\sum_{k=1}^{n-1} x_{k-1}^{2}}>\delta / 2 \text { for some } n \geq m\right\} .
\end{gathered}
$$

By the definition of $\tau(h)$ in (1.5)

$$
\begin{gathered}
\{\tau(h)<m\}=\left\{\sum_{k=1}^{m}\left(x_{k-1}^{2}+x_{k-2}^{2}\right)>h\right\} \\
=\left\{\sum_{k=1}^{m}\left(x_{k-1}^{2}+x_{k-2}^{2}\right)>h, \max _{1 \leq j \leq m}\left(x_{j-1}^{2}+x_{j-2}^{2}\right)<l\right\} \\
+\left\{\sum_{k=1}^{m}\left(x_{k-1}^{2}+x_{k-2}^{2}\right)>h, \max _{1 \leq j \leq m}\left(x_{j-1}^{2}+x_{j-2}^{2}\right) \geq l\right\} \\
\subset\{m l>h\} \cup \cup_{j=1}^{m}\left\{\left(x_{j-1}^{2}+x_{j-2}^{2}\right) \geq l\right\} .
\end{gathered}
$$

Therefore

$$
\mathbf{P}_{\theta}\{\tau(h)<m\} \leq I_{(m l>h)}+\sum_{k=1}^{m} \mathbf{P}_{\theta}\left\{x_{k-1}^{2}+x_{k-2}^{2} \geq l\right\}
$$


It remains to estimate the last term in the right-hand side of (3.17).

By the inequality

$$
\frac{\left\|M_{n}\right\|}{\sum_{k=1}^{n} x_{k-1}^{2}} \leq\left\|\frac{M_{n}}{\sum_{k=1}^{n} x_{k-1}^{2}}-L\left(\theta_{1}, \theta_{2}\right)\right\|+\left\|L\left(\theta_{1}, \theta_{2}\right)\right\|
$$

one has

$$
\begin{aligned}
& \mathbf{P}_{\theta}\left\{\frac{\left\|M_{n}\right\|}{\sum_{k=1}^{n} x_{k-1}^{2}} \cdot \frac{x_{n-1}^{2}}{\sum_{k=1}^{n-1} x_{k-1}^{2}}>\delta / 2 \text { for some } n \geq m\right\} \\
& \leq \mathbf{P}_{\theta}\left\{\left\|\frac{M_{n}}{\sum_{k=1}^{n} x_{k-1}^{2}}-L\left(\theta_{1}, \theta_{2}\right)\right\|>\sqrt{\delta / 4} \text { for some } n \geq m\right\} \\
& +\mathbf{P}_{\theta}\left\{L_{k}^{*} \frac{x_{n-1}^{2}}{\sum_{k=1}^{n-1} x_{k-1}^{2}}>\sqrt{\delta / 4} \text { for some } n \geq m\right\} \\
& +\mathbf{P}_{\theta}\left\{L_{k}^{*} \frac{x_{n-1}^{2}}{\sum_{k=1}^{n-1} x_{k-1}^{2}}>\delta / 4 \text { for some } n \geq m\right\},
\end{aligned}
$$

where $L_{k}^{*}=\sup _{\theta \in K}\left\|L\left(\theta_{1}, \theta_{2}\right)\right\|$.

Combining (3.19) $-(3.21)$ yields

$$
\begin{gathered}
\sup _{\theta \in K} \mathbf{P}_{\theta}\left(\left\|\frac{M_{\tau(h)}}{h}-L\left(\theta_{1}, \theta_{2}\right)\right\|>\delta\right) \\
\leq 2 I_{(m l>h)}+2 \sum_{k=1}^{m} \sup _{\theta \in K} \mathbf{P}_{\theta}\left\{x_{k-1}^{2}+x_{k-2}^{2} \geq l\right\} \\
+\mathbf{P}_{\theta}\left\{\left\|\frac{M_{n}}{\sum_{k=1}^{n} x_{k-1}^{2}}-L\left(\theta_{1}, \theta_{2}\right)\right\|>\frac{1}{2}(\delta \wedge \sqrt{\delta}) \text { for some } n \geq m\right\} \\
+\mathbf{P}_{\theta}\left\{L_{k}^{*} \frac{x_{n-1}^{2}}{\sum_{k=1}^{n-1} x_{k-1}^{2}}>\frac{1}{2}(\delta \wedge \sqrt{\delta}) \text { for some } n \geq m\right\} .
\end{gathered}
$$

Limiting $h \rightarrow \infty, l \rightarrow \infty, m \rightarrow \infty$ and taking into account Lemma 3.3 we come to (3.16). Hence Lemma 3.5.

Lemma 3.6 Let $x_{k}$ and $\tau(h)$ be defined by (1.1) and (1.5). Then for any compact set $K \subset \Lambda_{0}$ and $\delta>0$

$$
\lim _{h \rightarrow \infty} \sup _{\Theta \in K} P_{\Theta}\left(\frac{x_{\tau-1}^{2}}{\sum_{k=1}^{\tau-1} x_{k-1}^{2}}>\delta\right)=0 .
$$


Proof. One has the inclusion

$$
\left\{\frac{x_{\tau-1}^{2}}{\sum_{k=1}^{\tau-1} x_{k-1}^{2}}>\delta\right\} \subset\{\tau(h) \leq m\} \bigcup\left\{\frac{x_{n}^{2}}{\sum_{k=1}^{n-1} x_{k-1}^{2}}>\delta \text { for some } n \geq m\right\} \text {. }
$$

From here and (3.20) it follows that

$$
\begin{gathered}
\sup _{\Theta \in K} P_{\Theta}\left\{\frac{x_{\tau-1}^{2}}{\sum_{k=1}^{\tau-1} x_{k-1}^{2}}>\delta\right\} \leq I_{(m l>h)}+\sum_{j=1}^{m} \sup _{\Theta \in K} P_{\Theta}\left\{x_{j-1}^{2}+x_{j-2}^{2} \geq l\right\} \\
+\sup _{\Theta \in K} P_{\Theta}\left\{\frac{x_{n}^{2}}{\sum_{k=1}^{n-1} x_{k-1}^{2}}>\delta \text { for some } n \geq m\right\} .
\end{gathered}
$$

Limiting $h \rightarrow \infty, l \rightarrow \infty, m \rightarrow \infty$ and applying Lemma 3.2 lead to (3.22). This completes the proof of Lemma 3.6.

\section{Appendix.}

In this Section we prove some results used in the paper.

1. Proof of Lemma 2.1. Denote

$$
G_{\tau(h)}=L^{-1 / 2}\left(\theta_{1}, \theta_{2}\right) M_{\tau(h)} L^{-1 / 2}\left(\theta_{1}, \theta_{2}\right) .
$$

One can easily verify that

$$
\begin{gathered}
\left\|L^{-1 / 2}\left(\theta_{1}, \theta_{2}\right) \frac{M_{\tau(h)}^{1 / 2}}{\sqrt{h}}-I_{2}\right\|^{2}=\left\|\frac{1}{\sqrt{h}} G_{\tau(h)}^{1 / 2}-I_{2}\right\|^{2} \\
\leq\left\|h^{-1} G_{\tau(h)}-I_{2}\right\|^{2} \leq \operatorname{tr} L^{-1}\left(\theta_{1}, \theta_{2}\right)\left\|\frac{M_{\tau(h)}}{h}-L\left(\theta_{1}, \theta_{2}\right)\right\|^{2} .
\end{gathered}
$$

From here by Lemma 3.5 we come to the desired result.

Hence Lemma 2.1.

2. Proof of Lemma 2.2. We have

$$
v^{\prime} Y_{h}=\frac{1}{\sqrt{h}} \sum_{k=1}^{\tau(h)} g_{k-1} \varepsilon_{k}, g_{k-1}=v^{\prime} L^{-1 / 2}\left(\theta_{1}, \theta_{2}\right) X_{k-1} .
$$

For each $h>0$ we define the stopping time as

$$
\tau_{0}=\tau_{0}(h)=\inf \left\{n \geq 1: \sum_{k=1}^{n} g_{k-1}^{2} \geq h\right\}, \inf \{\emptyset\}=+\infty .
$$


Further we use the representation

$$
v^{\prime} Y_{h}=\frac{1}{\sqrt{h}} \sum_{k=1}^{\tau_{0}(h)} g_{k-1} \varepsilon_{k}+\eta(h)+\Delta(h)
$$

where $\Delta(h)=\Delta_{1}(h)+\cdots+\Delta_{4}(h)$,

$$
\begin{gathered}
\Delta_{1}(h)=h^{-1 / 2} I_{(\tau(h)=1)} g_{0} \varepsilon_{1}, \Delta_{2}(h)=h^{-1 / 2} g_{\tau(h)-1} \varepsilon_{\tau(h)}, \\
\Delta_{3}(h)=-h^{-1 / 2} I_{\left(\tau_{0}(h)=1\right)} g_{0} \varepsilon_{1}, \Delta_{4}(h)=-h^{-1 / 2} g_{\tau_{0}(h)-1} \varepsilon_{\tau_{0}(h)}, \\
\eta(h)=\frac{1}{\sqrt{h}} \sum_{k=1}^{\tau(h)-1} g_{k-1} \varepsilon_{k}-\frac{1}{\sqrt{h}} \sum_{k=1}^{\tau_{0}(h)-1} I_{\left(\tau_{0}>1\right)} g_{k-1} \varepsilon_{k} .
\end{gathered}
$$

Now we show that

$$
\begin{gathered}
\lim _{h \rightarrow \infty} \sup _{\theta \in K} \sup _{t \in R}\left|\mathbf{P}_{\theta}\left(\frac{1}{\sqrt{h}} \sum_{k=1}^{\tau_{0}(h)} g_{k-1} \varepsilon_{k} \leq t\right)-\Phi(t)\right|=0, \\
\lim _{h \rightarrow \infty} \sup _{\theta \in K} \mathbf{P}_{\theta}(|\eta(h)|>\delta)=0, \\
\lim _{h \rightarrow \infty} \sup _{\theta \in K} \mathbf{P}_{\theta}(|\Delta(h)|>\delta)=0 .
\end{gathered}
$$

The proof of (4.1) is based on Proposition 3.1 in Lai and Siegmund (1983). To this end we have to verify only the condition $A_{6}$, that is for each $\delta>0$

$$
\lim _{m \rightarrow \infty} \sup _{\theta \in K} \mathbf{P}_{\theta}\left(g_{n}^{2} \geq \delta \sum_{k=1}^{n} g_{k-1}^{2} \text { for some } n \geq m\right)=0
$$

Conditions $A_{1}-A_{5}$ are evidently satisfied.

First we note that

$$
\sum_{k=1}^{n} g_{k-1}^{2}=\left(v^{\prime} L^{-1 / 2}\left(\frac{M_{n}}{\sum_{k=1}^{n} x_{k-1}^{2}}-L\right) L^{-1 / 2} v+1\right) \sum_{k=1}^{n} x_{k-1}^{2} .
$$

Therefore we have

$$
\begin{gathered}
\left\{g_{n}^{2} \geq \delta \sum_{k=1}^{n} g_{k-1}^{2} \text { for some } n \geq m\right\} \\
\subseteq\left\{\left\|X_{n}\right\|^{2} \geq \delta_{1} \sum_{k=1}^{n} g_{k-1}^{2} \text { for some } n \geq m\right\}
\end{gathered}
$$




$$
\begin{gathered}
=\left\{\left\|X_{n}\right\|^{2} \geq \delta_{1} \sum_{k=1}^{n} x_{k-1}^{2}\left[1+v^{\prime} L^{-1 / 2}\left(\frac{M_{n}}{\sum_{k=1}^{n} x_{k-1}^{2}}-L\right) L^{-1 / 2} v\right] \text { for some } n \geq m\right\} \\
\subseteq\left\{\left\|X_{n}\right\|^{2} \geq \delta_{1} \sum_{k=1}^{n} x_{k-1}^{2}\left[1-\left\|L^{-1 / 2} v\right\|^{2} \cdot\left\|\frac{M_{n}}{\sum_{k=1}^{n} x_{k-1}^{2}}-L\right\|\right] \text { for some } n \geq m\right\} \\
\subseteq\left\{\left\|X_{n}\right\|^{2} \geq \delta_{1} \sum_{k=1}^{n} x_{k-1}^{2}\left[1-a^{*}\left\|\frac{M_{n}}{\sum_{k=1}^{n} x_{k-1}^{2}}-L\right\|\right] \text { for some } n \geq m\right\} \\
\subset\left\{\left\|\frac{M_{n}}{\sum_{k=1}^{n} x_{k-1}^{2}}-L\right\| \geq \frac{1}{2 a^{*}} \text { for some } n \geq m\right\} \\
\cup\left\{\left\|X_{n}\right\|^{2}>\frac{\delta_{1}}{2} \sum_{k=1}^{n} x_{k-1}^{2} \text { for some } n \geq m\right\},
\end{gathered}
$$

where $\delta_{1}=\delta / a^{*}, a^{*}=\sup _{\theta \in K}\left\|v^{\prime} L^{-1 / 2}\right\|^{2}$. This in view of Lemmas $2.5,3.1$ leads to (4.1).

It will be observed that (4.4) enables one to prove (by the same argument as in Lemma 3.6) that, for any compact set $K \subset \Lambda_{1}$ and $\delta>0$,

$$
\lim _{h \rightarrow \infty} \sup _{\theta \in K} P_{\theta}\left(g_{\tau_{0}-1}^{2} / \sum_{k=1}^{\tau_{0}-1} g_{k-1}^{2}>\delta\right)=0 .
$$

Now we check (4.2). One can easily verify that

$$
\mathbf{E}_{\theta} \eta^{2}(h)=\mathbf{E}_{\theta} u(h), u(h)=\frac{1}{h}\left|\sum_{k=1}^{\tau(h)-1} g_{k-1}^{2}-\sum_{k=1}^{\tau_{0}(h)-1} g_{k-1}^{2}\right|
$$

The random variable $u(h)$ is uniformly bounded from above uniformly in $\theta \in K$ because

$$
\begin{gathered}
u(h) \leq \frac{1}{h} \sum_{k=1}^{\tau(h)-1} g_{k-1}^{2}+1=\frac{1}{h} v^{\prime} L^{-1 / 2} M_{\tau(h)-1} L^{-1 / 2} v+1 \\
\leq \frac{a^{*}}{h} \sum_{k=1}^{\tau(h)-1}\left\|X_{k-1}\right\|^{2}+1 \leq a^{*}+1 .
\end{gathered}
$$

Therefore it suffices to establish that for each $\delta>0$

$$
\lim _{h \rightarrow \infty} \sup _{\theta \in K} \mathbf{P}_{\theta}(u(h)>\delta)=0
$$


To this end one can use the following estimate

$$
\begin{gathered}
u(h)=\frac{1}{h}\left|v^{\prime} L^{-1 / 2} M_{\tau(h)-1} L^{-1 / 2} v-\sum_{k=1}^{\tau_{0}(h)-1} g_{k-1}^{2}\right| \\
=\mid \frac{\sum_{k=1}^{\tau(h)-1} x_{k-1}^{2}}{h} v^{\prime} L^{-1 / 2}\left(\frac{M_{\tau(h)-1}}{\sum_{k=1}^{\tau(h)-1} x_{k-1}^{2}}-L\right) L^{-1 / 2} v \\
+\frac{\sum_{k=1}^{\tau(h)-1} x_{k-1}^{2}}{h}-\frac{\sum_{k=1}^{\tau_{0}(h)-1} g_{k-1}^{2}}{h} \mid \\
\leq a^{*}\left\|\frac{M_{\tau(h)-1}}{\sum_{k=1}^{\tau(h)-1} x_{k-1}^{2}}-L\right\|+\frac{x_{\tau(h)-1}^{2}}{\sum_{k=1}^{\tau(h)-1} x_{k-1}^{2}}+\frac{g_{\tau_{0}(h)-1}^{2}}{\sum_{k=1}^{\tau_{0}(h)-1} g_{k-1}^{2}} .
\end{gathered}
$$

From here by making use of (3.16), (3.22) and (4.5) we come to (4.6) which in its turn implies (4.2).

By a similar argument one can check (4.3). This completes the proof of Lemma 2.2. 


\section{References}

[1] Ahtola, J.A. and Tiao, G.C. (1987) Distribution of least squares estimators of autoregressive parameters for a process with complex roots on the unit circle. J. Time Ser. Anal. 8, p. 1-14.

[2] Anderson, T.W. (1971) The statistical analysis of time series. John Wiley Sons, New York.

[3] Anscombe, F. (1953) Sequential estimation. J. Roy. Statist. Soc., Ser. $B, \mathbf{1 5}$, p. 1-21.

[4] Chan, N.H. and Wei, C.Z. (1988) Limiting distribution of least squares estimates of unstable autoregressive processes. Ann. Statist., 16, p. 367-401.

[5] Dickey, D.A. and Fuller, W.A. (1979) Distribution of the estimators for autoregressive time series with a unit root. J. Amer. Statist. Assoc., 74, p. $427-431$.

[6] Galtchouk, L. and Konev V. (2003a) On uniform asymptotic normality of sequential least squares estimators for the parameters in a stable AR(1). Sequential Anal. 22, p. 31-54.

[7] Galtchouk, L. and Konev V. (2003 $)$ On uniform asymptotic normality of sequential least squares estimators for the parameters in a stable AR(p). J. Multivariate Anal. ?, p. ?.

[8] Greenwood, P.E. and Shiryaev, A.N. (1992) Asymptotic minimaxity of a sequential estimator for a first order autoregressive model. Stochastic and Stochastics Rep., 38, 49-65.

[9] Konev, V.V. and Pergamenshchikov, S.M. (1996) Guaranteed parameter estimation on the basis of a generalized least-squares method. Theory Probab. Appl. 41, p. 765-784.

[10] Lai, T.L. and Siegmund, D. (1983) Fixed-accuracy estimation of an autoregressive parameter. Ann.Statist. 11, p. 478-485.

[11] Lai, T.L. and Wei, C.Z. (1983) Asymptotic properties of general autoregressive models and strong consistency of least squares estimates of their parameters. J. Multivariate Anal. 13, p. 1-23.

[12] Nyblon, J. (1985) On the least squares estimators for the unstable AR(2) models. Contributed Papers, 45th Session, ISI, p. 385-386. 
[13] Rao, M.M. (1978) Asymptotic distribution of an estimator of the boundary parameter of an unstable process. Ann.Statist. 6, p. 185-190.

[14] Shiryaev, A.N. and Spokoiny, V.G. (1997) On sequential estimation of an autoregressive parameter. Stochastic and Stochastics Rep., 60, p. 219-240.

[15] Sriram, T.N. (1987) Sequential estimation of the mean of a first order stationary autoregressive process. Ann.Statist., 15, p. 1079-1090.

[16] Sriram, T.N. (1988) Sequential estimation of the autoregressive parameter in a first order autoregressive process. Sequential Anal., 7, p. 53-74.

[17] Stein, C. (1945) A two-sample test for a linear hypothesis whose power is independent of the variance. Ann. Math. Statist. 16, p. 243-258.

[18] White, J.S. (1958) The limiting distribution of the serial correlation coefficient in the explosive case. Ann. Math. Statist. 29, p. 1188-1197. 